Original Article

\title{
FORMULATION AND EVALUATION OF ATORVASTATIN CALCIUM NANOCRYSTALS CONTAINING P-GLYCOPROTEIN INHIBITORS FOR ENHANCING ORAL DELIVERY
}

\author{
SARAH LABIB ${ }^{1 *}$, MOHAMED NASR ${ }^{2}$, SABRY BADAWY ${ }^{1}$ \\ ${ }^{1}$ Department of Pharmaceutics and Industrial Pharmacy, Faculty of Pharmacy, Misr International University, Cairo, Egypt, ${ }^{2}$ Department of \\ Pharmaceutics and Industrial Pharmacy, Faculty of Pharmacy, Helwan University, Cairo, Egypt \\ *Email: sara.labib@miuegypt.edu.eg
}

Received: 25 Jan 2021, Revised and Accepted: 20 Mar 2021

ABSTRACT

Objective: The main objective of this study was to develop atorvastatin calcium (ATR) as an oral drug delivery system for a P-glycoprotein (P-gp) substrate drug using different pharmaceutical excipients that inhibit P-glycoprotein and evaluate the influence of nanocrystals on the dissolution characteristics and bioavailability compared to the plain drug.

Methods: A nanosuspension was prepared by Solvent-antisolvent precipitation method using a solvent containing stabilizer that act as a p-gp inhibitor dissolved in distilled water as polyethylene glycol 300, polyethylene glycol 400 (PEG 300, PEG 400), tween 20 and tween 80 while the solvent selected for atorvastatin calcium was methanol. The concentrations were as follows: PEG 300 and $400=0.25 \% \mathrm{w} / \mathrm{v}$, tween 20 and $80=$ $0.75 \% \mathrm{v} / \mathrm{v}$. Nanocrystals were extracted from the suspension and characterized.

Results: Particle size of the drug was $1307 \pm 127.79 \mathrm{~nm}$ while the formulas prepared ranged from $223 \pm 17.67$ to $887 \pm 58.12 \mathrm{~nm}$. Pure ATR had a saturated solubility of $0.059 \pm 0.005 \mathrm{mg} / \mathrm{ml}$ and the prepared nanocrystals ranged from $0.32 \pm 0.021$ to $0.88 \pm 0.019 \mathrm{mg} / \mathrm{ml}$. The Percentage of drug released of plain atorvastatin calcium reached $41.49 \%$ while the formula ranged from 44.32 to $61.5 \%$. Both XRD and SEM discussed the degree of crystallinity as follows: $\mathrm{F} 1<\mathrm{F} 2<\mathrm{F} 4<\mathrm{F} 3<\mathrm{ATR}$.

Conclusion: $0.3 \%$ of PEG 300 and PEG 400 were not enough to formulate proper nanocrystals while $0.75 \%$ tween 20 and tween 80 achieved acceptable formulas. F4 which is prepared with tween 80 exhibited the highest enhancement in saturated solubility, dissolution rate and subsequently expected to have improved oral bioavailability.

Keywords: Atorvastatin Calcium, Nanocrystals, Antisolvent Precipitation Method, Solubility, Bioavailability, P-glycoprotein

(c) 2021 The Authors. Published by Innovare Academic Sciences Pvt Ltd. This is an open access article under the CC BY license (https://creativecommons.org/licenses/by/4.0/)

DOI: https://dx.doi.org/10.22159/ijcpr.2021v13i3.42087 Journal homepage: https://innovareacademics.in/journals/index.php/ijcpr

\section{INTRODUCTION}

$40 \%$ of pharmaceutical compounds are poorly water-soluble which affect the absorption and bioavailability of the drug [1] . Therefore enhancement of the extent of absorption for poorly aqueous soluble drugs intended for oral use is still one of the most critical and challenging approaches in the improvement of dosage forms [2].

One of the approaches to improve the solubility of the drug is to formulate it into nanocrystals which are crystalline particles with at least one dimension measuring less than 1000 nanometers [3]. Nanocrystal formulations can be either partially or completely crystalline depending on the method of formulation and those designed for oral administration have several advantages as the high rate of absorption and oral bioavailability, rapid action, improved dose proportionality, lower required dose and being compatible with all routes of administration in any dosage form [4].

The drug chosen for this study was atorvastatin calcium which is one of HMG CoA reductase inhibitors or statins that can reduce the levels of low-density lipoprotein and triglycerids in the blood while elevating the level of high-density lipoprotein [5]. Atorvastain is used to treat high cholesterol and to lower the risk of stroke, heart attack and other cardiac complications [5]. It is a class II drug with low aqueous solubility and high intestinal permeability but it is a pgp substrate drug, therefore it has a low bioavailability of $14 \%[6,7]$.

Permeability glycoprotein (p-gp) is an ATP-dependent efflux pump with broad substrate specificity extensively distributed and expressed in the intestinal epithelium, liver cells, and the capillary endothelial cells composing the blood-brain barrier and blood-testis barrier [8]. As a consequence of its tissue localization and its broad substrate specificity, P-gp plays a key role in the absorption, distribution, and elimination of many drugs thus reducing the bioavailability and explaining how a drug of high permeability like atorvastatin has a reduced oral bioavailability [9].

The objective of this study is to improve the oral bioavailability of atorvastatin calcium by two means. The first is enhancing the solubility by decreasing the particle size to the nano range and formulation of nanocrystals while the second is augmenting the intestinal absorption through preparing nanocrystals using stabilizers that act as p-gp inhibitors to decrease or eliminate the effect of intestinal efflux on the absorption.

\section{MATERIALS AND METHODS}

Atorvastatin Calcium was kindly gifted by Amoun Pharmaceuticals Co., Egypt. Tween 80 and tween 20 were purchased from Lab-scan analytical sciences, Poland. Polyethylene 300 and polyethylene 400 were obtained from Loba Chemie. Methanol HPLC grade was purchased from Sigma-Aldrich co., Germany.

\section{Prepration and extraction of nanocrystals}

A nanosuspension was prepared by Solvent-antisolvent precipitation method using a solvent: antisolvent ratio of 25:75 followed by extraction of nanocrystals [10]. The solvent selected to dissolve atorvastatin calcium (ATR) was methanol and supersaturation was achieved at a concentration of $60 \mathrm{mg} / \mathrm{ml}$. The antisolvent contained a hydrophilic stabilizer that act as a p-gp inhibitor dissolved in distilled water. The stabilizers chosen for this study were polyethylene glycol 300, polyethylene glycol 400 (PEG 300 ,PEG 400), tween 20 and tween 80 [11]. Each one of them was used to prepare an antisolvent with different concentrations and four formulas were evaluated in this study. The concentrations were as follows: PEG 300 and $400=0.3 \% \mathrm{w} / \mathrm{v}$, tween 20 and $80=0.75 \%$ $\mathrm{v} / \mathrm{v}$. The antisolvent was cooled in an ice bath and homogenized 
using a mechanical stirrer then the solvent containing the drug was added drop wise. The blend was homogenized for 1 hour. Extraction of the nanocrystals was achieved by solvent evaporation after being left for $24 \mathrm{~h}$ to dry at room $\left(25^{\circ} \mathrm{C}\right)$.

\section{Characterization of formulas}

\section{Particle size analysis}

The mean particle size and polydispersity index (Pdi) were measured at $25 \pm 0.5^{\circ} \mathrm{C}$ at a measuring angle of $90^{\circ}$ to the incident beam using Zeta Sizer Nano-ZS, Malvern, UK. ATR powder and nanocrystals were prepared in triplicate by dispersing in deionized water, sonicated for $10 \mathrm{~min}$ and filtered before assessment [12].

\section{Scanning electron microscopy (SEM)}

A scanning electron microscope (JEOL JSM-5500 LV-JEOL Ltd, Japan) was used to obtain images of the drug and formulas to study their morphology by using high vacuum mode at an accelerating voltage of $20 \mathrm{kV}$. The samples were coated by a gold sputter coater (SPI-Module) and fixed on brass stub using adhesive tape prior to observation [13].

\section{Powder X-ray diffractometry (PXRD)}

For further confirmation of the physical state of the drug and formulas, X-ray diffraction patterns were obtained using the X-ray diffractometer (X'Pert-PRO Diffractometer, PANalytical, the Netherlands) with $\mathrm{Cu}$ as tube anode. The following conditions were used to record the diffractograms, the voltage was $45 \mathrm{kV}$, steps were $0.02^{\circ}$ of $\left({ }^{\circ} 2 \theta\right.$ ), data were collected from 0 to $70^{\circ} 2 \theta$, and the counting rate was $0.5 \mathrm{~s} / \mathrm{step}$ at room temperature [14].

\section{Saturated solubility}

Saturated solubility was achieved by adding an excess amount of samples to $50 \mathrm{ml}$ phosphate buffer solution ( $\mathrm{pH}$ 6.8). The solutions were fixed on a shaking water bath for $48 \mathrm{~h}$ at $37 \pm 0.5^{\circ} \mathrm{C}$ until equilibrium was attained [15]. The equilibrated samples were centrifuged at $5,000 \mathrm{rpm}$ for $5 \mathrm{~min}$. After decantation, the supernatants were filtered through $0.45 \mu \mathrm{m}$ membrane filter, diluted and assayed using a UV-visible spectrophotometer against a blank at $246 \mathrm{~nm}$ [16]. Every sample was analyzed in triplicate and the mean values and standard deviations were reported

\section{In vitro dissolution test}

The dissolution studies were performed according to the paddle method (USP) operated at 100rpm using Hanson dissolution apparatus (Hanson Research, California, USA). The dissolution medium was $900 \mathrm{ml}$ of phosphate buffer ( $\mathrm{pH} \mathrm{6.8)}$, maintained at 37 ${ }^{\circ} \mathrm{C} \pm 0.5{ }^{\circ} \mathrm{C}$ [17]. Samples containing the equivalent of $20 \mathrm{mg}$ ATR were dispersed in the dissolution medium. $3 \mathrm{ml}$ were withdrawn at different time intervals, filtered through a $0.45 \mu \mathrm{m}$ membrane filter, suitably diluted, and analyzed using a spectrophotometer for ATR content at $246 \mathrm{~nm}$ [18]. Withdrawn samples were compensated by fresh medium. The dissolution experiments were conducted in triplicate and calculated mean values of cumulative drug release were used to plot the release curve.

\section{RESULTS AND DISCUSSION}

\section{Particle size analysis}

Particle size of the drug was $1307 \pm 127.79 \mathrm{~nm}$ while the formulas prepared ranged from $223 \pm 17.67$ to $887 \pm 58.12 \mathrm{~nm}$. According to the results mentioned in table 1 , nanoparticles were formed and their particle size was significantly reduced relative to that of the drug ( $P$ 0.05 ), which proves that the method of preparation and extraction of this study was successful. Samples formulated using tween 80 and 20 had smaller particle size and lower PDI than PEG 300 and PEG 400. PDI of F1 and F2 is lower than 0.5 indicating that the nanoparticle formulation has a high level of distribution homogeneity unlike F3 and F4 [19]. according to both parameters, $\mathrm{F} 1$ and F2 are more promising.

Table 1: Particle size, polydispersity index and saturated solubility

\begin{tabular}{lllll}
\hline Formulas code & Stabilizer used & Mean particle size(nm \pm SD,n=3) & PDI & Saturated solubility in mg/ml (mean \pm SD,n=3) \\
\hline ATR & Pure drug & $1307 \pm 127.79$ & 0.985 & $0.059 \pm 0.005$ \\
F1 & PEG 300 & $887 \pm 58.12$ & 0.756 & $0.31 \pm 0.036$ \\
F2 & PEG 400 & $753 \pm 67.51$ & 0.732 & $0.42 \pm 0.021$ \\
F3 & T20 & $341 \pm 29.34$ & 0.389 & $0.59 \pm 0.050$ \\
F4 & T80 & $223 \pm 17.67$ & 0.204 & $0.88 \pm 0.019$ \\
\hline
\end{tabular}

\section{Saturated solubility}

Results of the saturated solubility studies are indicated in table 1. Pure ATR had a saturated solubility of $0.059 \pm 0.005 \mathrm{mg} / \mathrm{ml}$ and the prepared nanocrystals ranged from $0.32 \pm 0.021$ to $0.88 \pm 0.019$ $\mathrm{mg} / \mathrm{ml}$. The saturated solubility of all formulas increased significantly $(\mathrm{P}<0.05)$ compared to plain drugs. This result may to be due to the significant particle size reduction [20]. F4 showed the lowest particle size and highest saturated solubility $(0.88 \pm 0.019$ $\mathrm{mg} / \mathrm{ml}$ ) compared to the other formulas which confirm that particle size is inversely proportional to solubility [21].

\section{Scanning electron microscopy (SEM)}

The shape and surface characteristics of ATR and nanocrystals were visualized using SEM as in fig. 1. Plain Atorvastatin calcium, F3 and F4 have uniform rod-shaped crystals which confirm the success of the antisolvent precipitation method while F1 and F2 showed irregular aggregated particles especially F1, also high degree of tackiness was observed after extraction from nanosuspension, unlike F3 and F4 that yielded free-flowing fine powder. The aggregation and tackiness of F1 and F2 may be caused by the low affinity of PEG 300 and PEG 400 to the newly formed crystal surface or their concentration of $(0.3 \%)$ was not sufficient to form a protective layer on the crystal surface reduce or prevent stickiness of the crystals formed [14]. This finding suggest that The higher particle size of both F1 and F2 might be attributed to the aggregation rather than to the crystal growth [22]. Also, possible transformation into an amorphous form is suspected in F1, it might be confirmed or denied by the XRD analysis.

\section{In vitro dissolution test}

10 samples of each formula were taken in different time intervals in the course of $120 \mathrm{~min}$ and the percentage of drug released was calculated. All formulas exhibited higher dissolution characteristics than atorvastatin calcium in phosphate buffer PH (6.8) as shown in table 2 . the plain atorvastatin calcium reached $41.49 \%$ while the formula ranged from 44.32 to $61.5 \%$.

It was also observed that the cumulative percentage of drug released in F1 and F2 were not significantly higher than drug $(\mathrm{P}<0.05)$ and they took a longer time to dissolve completely proofing that the aggregation hindered the dissolution [23]. These findings indicate that $0.3 \%$ of PEG 300 and PEG 400 were not sufficient to achieve better formulas. The dissolution profiles of F3 and F4 showed a significantly improved percentage of drug release and rapid dissolving of powder in the medium [24]. According to this data, F4 is the best formula.

\section{Powder X-ray diffractometry (PXRD)}

In fig. $3 \mathrm{X}$-ray diffraction patterns of drug and formulas were discussed to investigate the presence of any polymorphic transformation. The diffractogram of pure atorvastatin calcium shows sharp characteristic peaks of high intensity which proofs its crystalline nature. Characteristic peaks were spotted at intensity reflection counts of $310.46,433.3,750.5$ and 358.77 at diffraction angles of $(2 \theta) 16.8739^{\circ}$, $19.2897^{\circ}, 21.4145^{\circ}$ and $23.5345^{\circ}$ respectively. These peaks are present in the diffractograms of F3 and F4 at the same diffraction angles but with the decrease in the reflections counts revealing a reduction in 
their crystallinity. In F3 peaks are sharp like the drug with a slight decrease in intensity while in F4 the sharpness decreased more as well as their intensity indicating that F3 is slightly lower in crystallinty while F4 is even lower. Drugs with lower crystallinity and smaller particle size have a higher dissolution rate and bioavailability which explains why F4 has the highest solubility and dissolution rate [25, 26]. Distinct decrease in peaks intensity was observed in F2 and some peaks were absent which is attributed to the partial transformation into amorphous form and could be due to the tackiness and aggregation present in the formula as observed in the SEM image. The characteristic crystalline peaks in F1 appeared broadened and almost distorted as compared to the pure drug confirming the possible transformation into an amorphous form which was suspected from the SEM image and the aggregation that occurred during the preparation of the formula [26]. Despite having a lower level of crystallinity the dissolution wasn't significantly higher which gives further confirmation that aggregation hinders and lowers the solubility and dissolution of formulas.
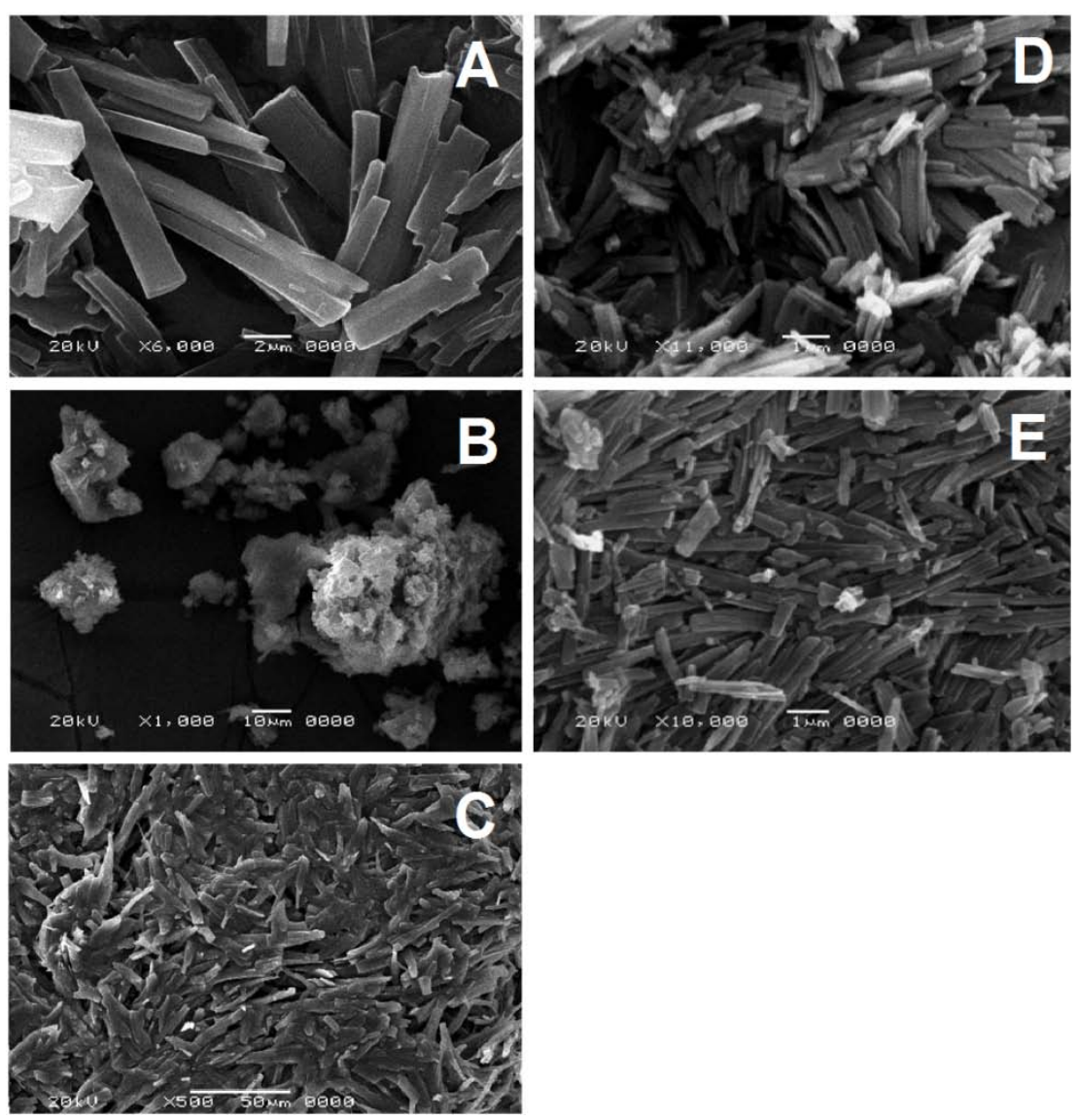

Fig. 1: SEM images of (A) plain atorvastatin, (B) F1, (C)F2, (D)F3, (E)F4

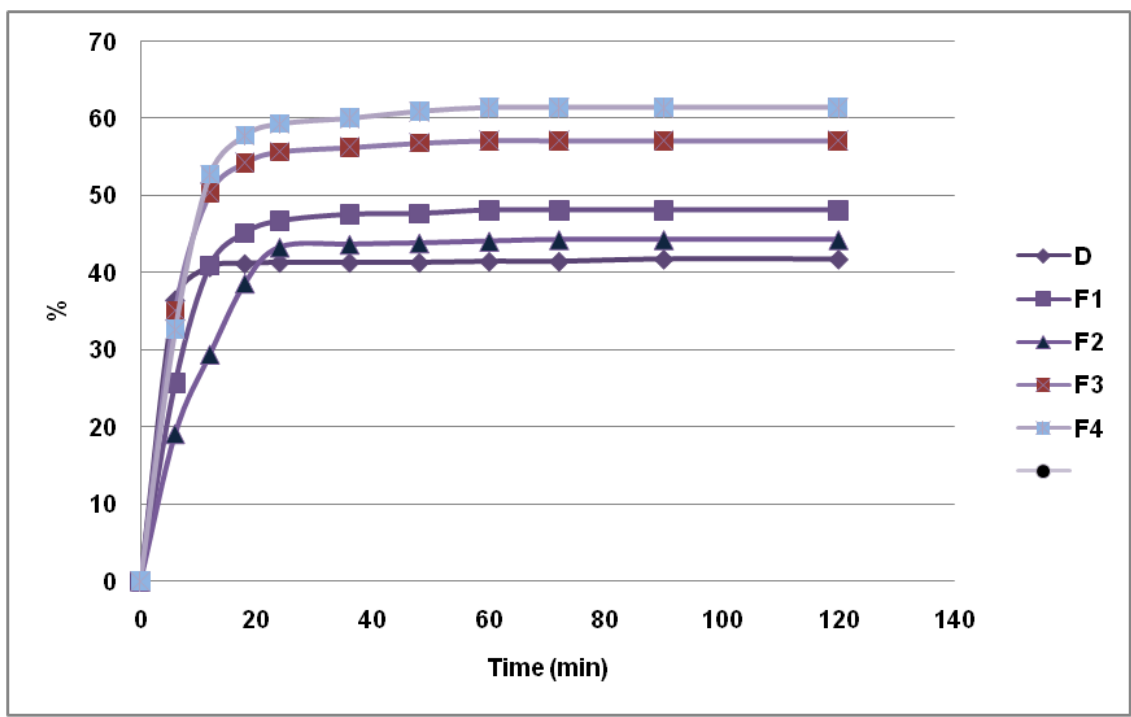

Fig. 2: In vitro drug release profiles of (D) drug and formulas in phosphate buffer pH 6.8 
Table 2: In vitro drug release profiles of (D) drug and formulas in phosphate buffer pH 6.8

\begin{tabular}{|c|c|c|c|c|c|}
\hline \multicolumn{6}{|c|}{ Cumulative \% of drug released } \\
\hline Time (min) & D & F1 & F2 & F3 & F4 \\
\hline 0 & 0 & 0 & 0 & 0 & 0 \\
\hline 6 & 36.5 & 19.17 & 25.76 & 35.12 & 32.67 \\
\hline 12 & 40.77 & 29.48 & 40.98 & 50.42 & 52.8 \\
\hline 18 & 41.2 & 38.67 & 45.18 & 54.31 & 57.86 \\
\hline 24 & 41.35 & 43.35 & 46.72 & 55.75 & 59.37 \\
\hline 36 & 41.35 & 43.71 & 47.56 & 56.32 & 60.1 \\
\hline 48 & 41.35 & 43.88 & 47.68 & 56.89 & 61 \\
\hline 60 & 41.49 & 44.12 & 48.13 & 57.2 & 61.5 \\
\hline 72 & 41.49 & 44.32 & 48.13 & 57.2 & 61.5 \\
\hline 90 & 41.78 & 44.32 & 48.13 & 57.2 & 61.5 \\
\hline 120 & 41.78 & 44.32 & 48.13 & 57.2 & 61.5 \\
\hline
\end{tabular}

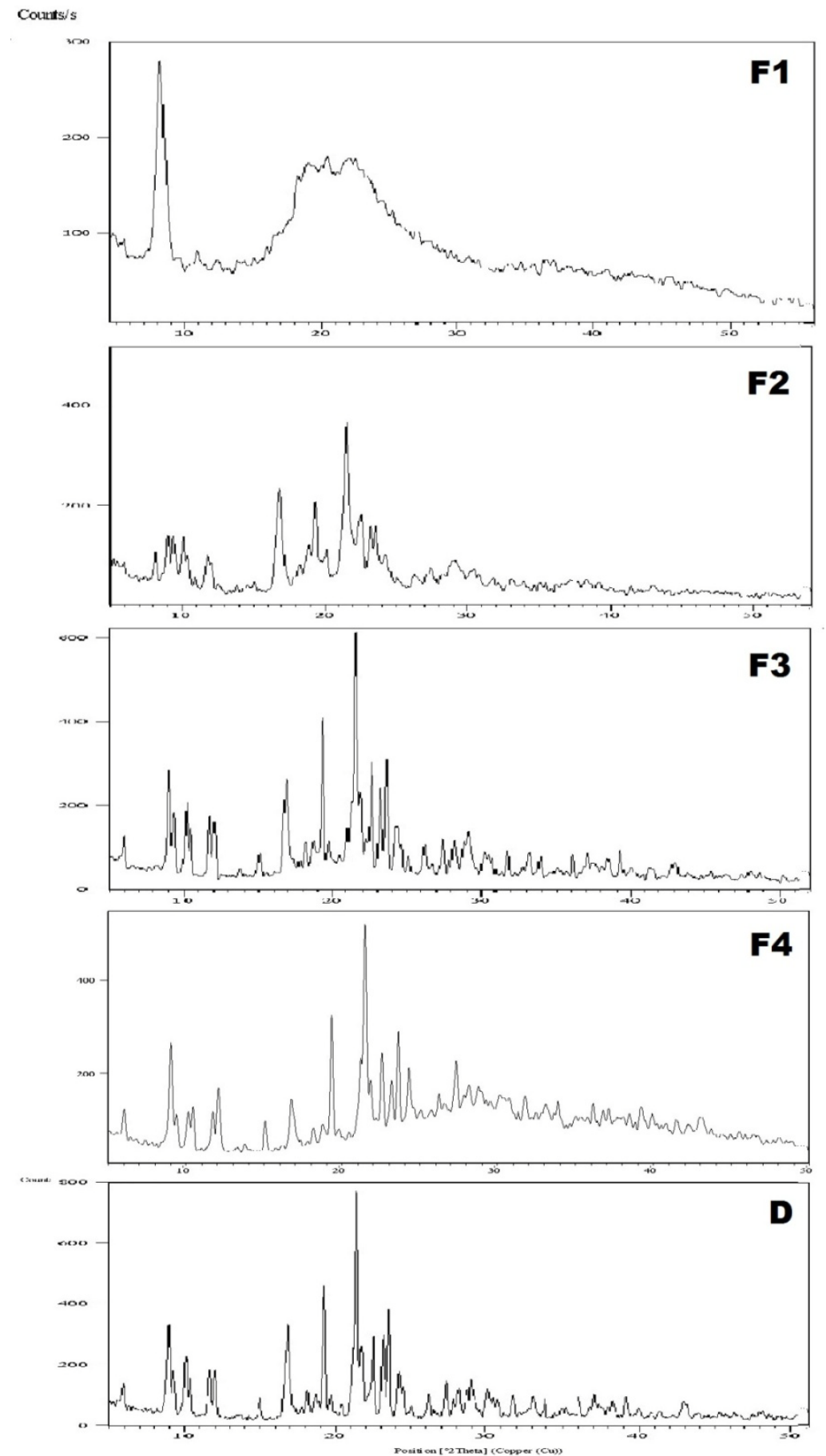

Fig. 3: XRD patterns of (D)plain drug, (F1) formula prepared PEG 300, (F2) byPEG 400, (F3) by tween 20,(F4) by tween 80 


\section{CONCLUSION}

The method of preparation used was successful to form nanoparticles as shown in the particle siz study but the formulas produced have differnet degrees of crystallinty. the XRD analysis illusterted that the degree of crystallinty was arranged as follows, F1 $<$ F2 $<$ F $4<F 3<$ ATR. although lower crysallinty means higher solubility and disolution, they were arranged as follows $\mathrm{ATR}<\mathrm{F} 1<\mathrm{F} 2<\mathrm{F} 3<\mathrm{F} 4$. It was expected for F1 and F2 to have a higher dissolution rate but they were the least due to the tackiness and aggregation as well as the larger particle size. both F3 and F4 were significantly enhanced but F4 was the best formula in terms of the degree of saturated solubility and dissolution. it may be attributed to the lower crystallinity of $\mathrm{F} 4$, the absence of aggregation and having the lowest particle size. To sum up, 0.3\%PEG 300 and PEG 400 were not sufficient to form a protective barrier on the newly formed nanocrystals so preparing formulas with higher concentration was considered and the best formula selected from this study is F4 which is prepared using $0.75 \%$ tween 80 as a stabilizer as it showed a marked enhancement in dissolution and subsequently in bioavailability.

\section{FUNDING}

Nil

\section{AUTHORS CONTRIBUTIONS}

All the authors have contributed equally.

\section{CONFLICT OF INTERESTS}

The authors report no conflicts of interest.

\section{REFERENCES}

1. Maiti SJJoPST. Liquid-crystal and nano-crystal technology for solubilization of poorly water-soluble drugs. J PharmSciTech 2012;2:1-4.

2. Sareen S, Mathew G, Joseph LJIjopi. Improvement in solubility of poor water-soluble drugs by solid dispersion. Int J Pharm Investig 2012;2:12-7.

3. Zhang L, Webster TJJNt. Nanotechnology and nanomaterials: promises for improved tissue regeneration. Nano today 2009;4:66-80.

4. Desu PK, Sindhuja M, Thriveni K, Nagalakshmi V, Rao PVJWJPPS. A review on the significance of nanocrystals in drug delivery. World J Pharm Pharm Sci 2017;6:347-58.

5. Koh KK, Quon MJ, Waclawiw MAJA. Are statins effective for simultaneously treating dyslipidemias and hypertension? Atherosclerosis 2008;196:1-8.

6. Elmowafy M, Ibrahim H, Ahmed M, Shalaby K, Salama A, Hefesha H. Atorvastatin-loaded nanostructured lipid carriers (NLCs): strategy to overcome oral delivery drawbacks. Drug Delivery 2017;24:932-41.

7. Khan FN, Dehghan MHGJAP. Enhanced bioavailability of atorvastatin calcium from the stabilized gastric resident formulation. AAPS PharmSciTech 2011;12:1077-86.

8. Thiebaut F, Tsuruo T, Hamada H, Gottesman MM, Pastan I, Willingham MCJPotNAoS. Cellular localization of the multidrugresistance gene product P-glycoprotein in normal human tissues. Proc Natl Acad Sci USA 1987;84:7735-8.

9. Leveque D, Jehl FJAr. P-glycoprotein and pharmacokinetics. Anticancer Res 1995;15:331-6.

10. de Paiva Lacerda S, Espitalier F, Hoffart V, Re MIJDd, Pharmacy I. Liquid anti-solvent recrystallization to enhance dissolution of
CRS 74, a new antiretroviral drug. Drug Dev Industrial Pharm 2015;41:1910-20.

11. Zhang H, Yao M, Morrison RA, Chong SJAopr. Commonly used surfactant, Tween 80, improves absorption of P-glycoprotein substrate, digoxin, in rats. 2003;26:768-72.

12. Chin SF, Mohd Yazid SNA, Pang SCJIJoPS. Preparation and characterization of starch nanoparticles for controlled release of curcumin. Int J Polymer Sci 2014. https://doi.org/10.1155/2014/340121

13. Jukanti R, Sheela S, Bandari S, Veerareddy PRJJops. Enhanced bioavailability of exemestane via proliposomes based transdermal delivery. J Pharm Sci 2011;100:3208-22.

14. Nasr MJAP. Influence of microcrystal formulation on in vivo absorption of celecoxib in rats. AAPS PharmSciTech 2013;14:719-26.

15. Rodde MS, Divase GT, Devkar TB, Tekade ARJBri. Solubility and bioavailability enhancement of poorly aqueous soluble atorvastatin: in vitro, ex vivo, and in vivo studies. Biomed Res Int 2014. DOI:10.1155/2014/463895

16. Hecq J, Deleers M, Fanara D, Vranckx H, Amighi KJIjop. Preparation and characterization of nanocrystals for solubility and dissolution rate enhancement of nifedipine. Int J Pharm 2005;299:167-77.

17. Rahat Jahan M, Islam S, Tanwir A, Chowdhury JAJJoapt, Research. In vitro dissolution study of atorvastatin binary solid dispersion. J Adv Pharm Technol Res 2013;4:18.

18. Arunkumar N, Deecaraman M, Rani C, Mohanraj K, Kumar KVJIJPTR. Preparation and solid-state characterization of atorvastatin nanosuspensions for enhanced solubility and dissolution. Int J PharmTech Res 2009;1:1725-30.

19. Masarudin MJ, Cutts SM, Evison BJ, Phillips DR, Pigram PJJN Science, Applications. Factors determining the stability, size distribution, and cellular accumulation of small, monodisperse chitosan nanoparticles as candidate vectors for anticancer drug delivery: application to the passive encapsulation of ${ }^{14} \mathrm{C}$ doxorubicin. Nanotechnol Sci Appl 2015;8:67.

20. Choudhary A, Rana AC, Aggarwal G, Kumar V, Zakir FJAPSB. Development and characterization of an atorvastatin solid dispersion formulation using skimmed milk for improved oral bioavailability. Acta Pharm Sin B 2012;2:421-8.

21. Behera A, Sahoo S, Patil SJDPL. Enhancement of solubility: a pharmaceutical overview. Pharm Lett 2010;2:310-8.

22. Rasenack N, Müller BWJPr. Dissolution rate enhancement by in situ micronization of poorly water-soluble drugs. Pharm Res 2002;19:1894-900

23. Sharma M, Mehta I. Surface stabilized atorvastatin nanocrystals with improved bioavailability, safety and antihyperlipidemic potential. Scientific Reports 2019;9:16105.

24. Panghal D, Nagpal M, Thakur GS, Arora SJSp. Dissolution improvement of atorvastatin calcium using modified locust bean gum by the solid dispersion technique. Pharm Res 2014;82:177-92.

25. Zhong J, Shen Z, Yang Y, Chen JJIjop. Preparation and characterization of uniform nanosized cephradine by the combination of reactive precipitation and liquid anti-solvent precipitation under high gravity environment. Int J Pharm 2005;301:286-93.

26. Zhang HX, Wang JX, Zhang ZB, Le Y, Shen ZG, Chen JFJIjop. Micronization of atorvastatin calcium by antisolvent precipitation process. Int J Pharm 2009;374:106-13. 